\title{
The Higgs and leptophobic force at the LHC
}

\author{
Pavel Fileviez Pérez, ${ }^{a}$ Elliot Golias, ${ }^{a}$ Clara Murgui ${ }^{b}$ and Alexis D. Plascencia ${ }^{a}$ \\ ${ }^{a}$ Physics Department and \\ Center for Education and Research in Cosmology and Astrophysics (CERCA), \\ Case Western Reserve University, Cleveland, OH 44106, U.S.A. \\ ${ }^{b}$ Departamento de Física Teórica, IFIC, Universitat de Valencia-CSIC, \\ Valencia E-46071, Spain \\ E-mail: pxf112@case.edu, ebg23@case.edu, clara.murgui@ific.uv.es, \\ alexis.plascencia@case.edu
}

ABstract: The Higgs boson could provide the key to discover new physics at the Large Hadron Collider. We investigate novel decays of the Standard Model (SM) Higgs boson into leptophobic gauge bosons which can be light in agreement with all experimental constraints. We study the associated production of the SM Higgs and the leptophobic gauge boson that could be crucial to test the existence of a leptophobic force. Our results demonstrate that it is possible to have a simple gauge extension of the SM at the low scale, without assuming very small couplings and in agreement with all the experimental bounds that can be probed at the LHC.

Keywords: Beyond Standard Model, Higgs Physics

ARXIV EPRINT: 2003.09426 


\section{Contents}

1 Introduction $\quad 1$

2 Leptophobic gauge boson at the LHC 2

3 Exotic decays of the SM-like Higgs 4

4 Higgs-leptophobic gauge boson associated production $\quad 6$

$\begin{array}{llr}5 & \text { Summary } & 8\end{array}$

$\begin{array}{ll}\text { A Decays widths } & 10\end{array}$

$\begin{array}{ll}\text { B Production cross-sections } & 11\end{array}$

$\begin{array}{ll}\text { C Constraints from kinetic mixing } & 14\end{array}$

\section{Introduction}

The discovery of the Standard Model (SM) Higgs boson with a mass of $125 \mathrm{GeV}$ at the Large Hadron Collider (LHC) [1, 2] can be considered one of the most important discoveries in physics. We now understand how most of the elementary particles acquire mass through the Higgs mechanism and how the electroweak symmetry is spontaneously broken in nature. Thanks to the great effort of the experimental collaborations at the LHC we know well the properties of the SM Higgs and there exist experimental constraints on its decays and production mechanisms, see for example ref. [3] for a detailed discussion.

The Higgs boson could open a door to a new physics sector since it can have new interactions that can provide information about a theory for physics beyond the Standard Model. The LHC could discover new decays and/or production channels for the Higgs boson and combining different searches we could have access to new interactions and discover new particles with masses below the TeV scale. See ref. [4] for a report on future studies at the LHC.

In this article, we investigate new possible decays and production mechanisms of the Higgs boson due to the existence of a new interaction with a leptophobic gauge boson. A leptophobic gauge boson is predicted in simple theories where baryon number is a local gauge symmetry [5-8] spontaneously broken at the low scale. See refs. [9-11] for realistic models predicting a leptophobic gauge boson and ref. [12] for a review. In our studies we show that one can have a large branching ratio for the Higgs decays into two leptophobic gauge bosons if they are kinematically allowed. The leptophobic gauge boson can be light 
with mass below the electroweak scale in agreement with all experimental bounds and without assuming a very small gauge coupling.

When the new Higgs decays are highly suppressed or not allowed we investigate the associated Higgs-leptophobic gauge boson production mechanism at the LHC. We find that, using this production mechanism, one can obtain large number of events with multiphotons and two quarks that can be used to test the existence of a new interaction of the Higgs boson with this new gauge boson. As in the case of the Higgs decays, the production cross-sections can be generically large due to the fact that the leptophobic gauge boson can be light in agreement with all experimental bounds. The possible existence of a leptophobic gauge boson at the low scale tells us that a gauge theory where baryon number is a local symmetry [8-11] can describe physics below the TeV scale.

This article is organized as follows: in section 2, we review all current collider constraints on a leptophobic gauge boson and discuss the impact of these bounds on the predictions for production cross-sections at the LHC. In section 3, we show the predictions for the new Higgs decay channels into two leptophobic gauge bosons taking into account all the experimental constraints. In section 4 , we discuss the associated production channel proton-proton to the leptophobic gauge boson and the SM Higgs, $p p \rightarrow Z_{B}^{*} \rightarrow Z_{B} h$, and investigate the different signatures at the LHC. We present our conclusions in section 5 . Appendices A and B contain analytic results for all the processes considered in this work. In appendix $\mathrm{C}$, we discuss the bounds on the kinetic mixing between the $Z$ and the new gauge boson.

\section{Leptophobic gauge boson at the LHC}

In simple extensions of the SM where baryon number is a local symmetry [8-11] spontaneously broken one predicts the existence of a leptophobic gauge boson $Z_{B}$. For phenomenological studies of these models and dark matter see refs. [13-17], while for a mechanism for baryogenesis in this scenario see ref. [18]. The coupling between the SM quarks and $Z_{B}$ in our convention is given by

$$
Z_{B}^{\mu} \bar{q} q:-i \frac{g_{B}}{3} \gamma^{\mu}
$$

As we show in the following, the local baryon number can be broken at the low scale, even at energies below the electroweak scale.

The main strategy to search for a heavy $Z_{B}$ at the LHC is by looking for a dijet resonance. However, at low masses this search loses sensitivity due to the large QCD backgrounds. Nonetheless, recent experimental searches for a boosted leptophobic gauge boson decaying into jets along with initial state radiation of a photon have been performed at CMS to place exclusion bounds down to a mass of $10 \mathrm{GeV}$ for $Z_{B}$ [19]. This further motivates a study in the low mass region.

In figure 1 we summarize the current collider bounds for the leptophobic gauge boson in the $g_{B}-M_{Z_{B}}$ plane. As this figure shows, there is a large region in the parameter space that remains unconstrained. Specifically, for a light $Z_{B}$ with mass between 25 and $50 \mathrm{GeV}$ the gauge coupling can take relatively large values. For smaller couplings, i.e. $g_{B} \lesssim 0.1$, 

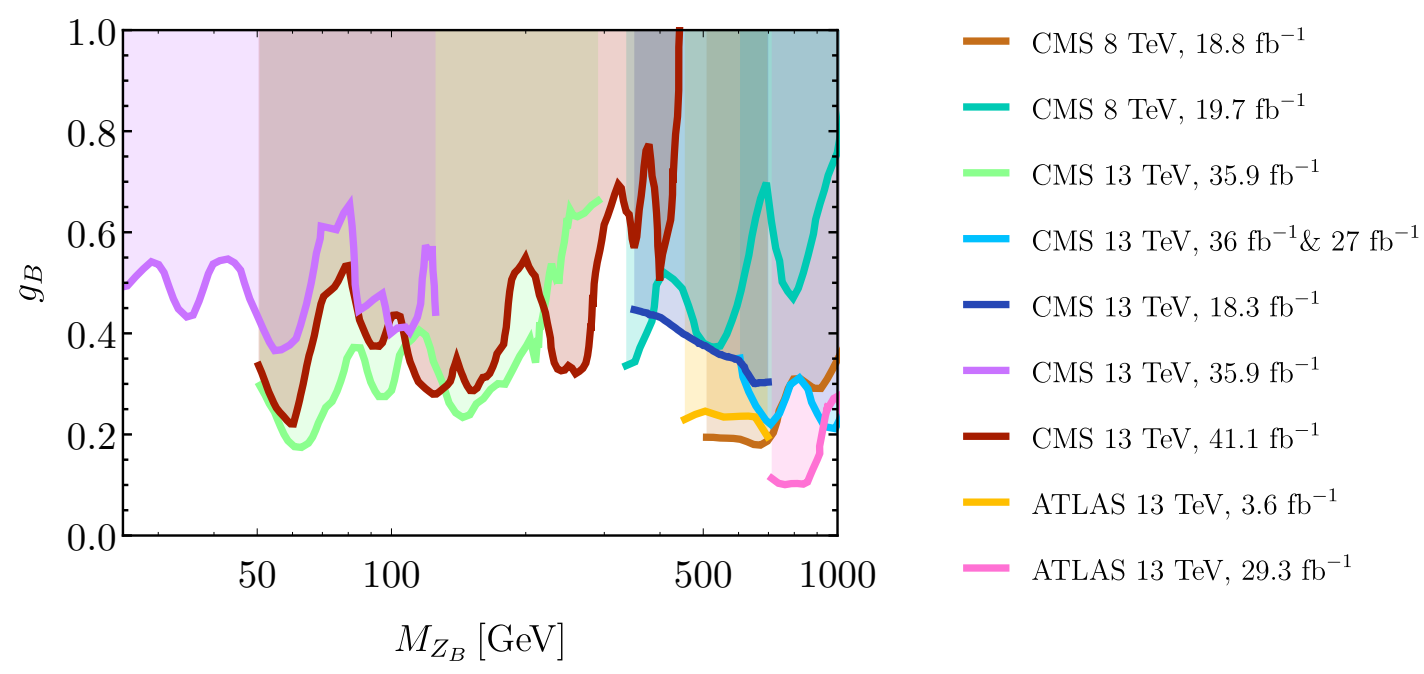

Figure 1. Experimental bounds for the leptophobic gauge boson $Z_{B}$. Here, we use the CMS analyses ( $8 \mathrm{TeV}$ and $18.8 \mathrm{fb}^{-1}[20], 8 \mathrm{TeV}$ and $19.7 \mathrm{fb}^{-1}[21], 13 \mathrm{TeV}$ and $35.9 \mathrm{fb}^{-1}[19,22]$ and $41.1 \mathrm{fb}^{-1}[22], 13 \mathrm{TeV}$ and $36 \mathrm{fb}^{-1} \& 27 \mathrm{fb}^{-1}$ [23], $13 \mathrm{TeV}$ and $18.3 \mathrm{fb}^{-1}$ [24]), and ATLAS results $\left(13 \mathrm{TeV}\right.$ and $3.6 \mathrm{fb}^{-1}$ and $\left.29.3 \mathrm{fb}^{-1}[25]\right)$.
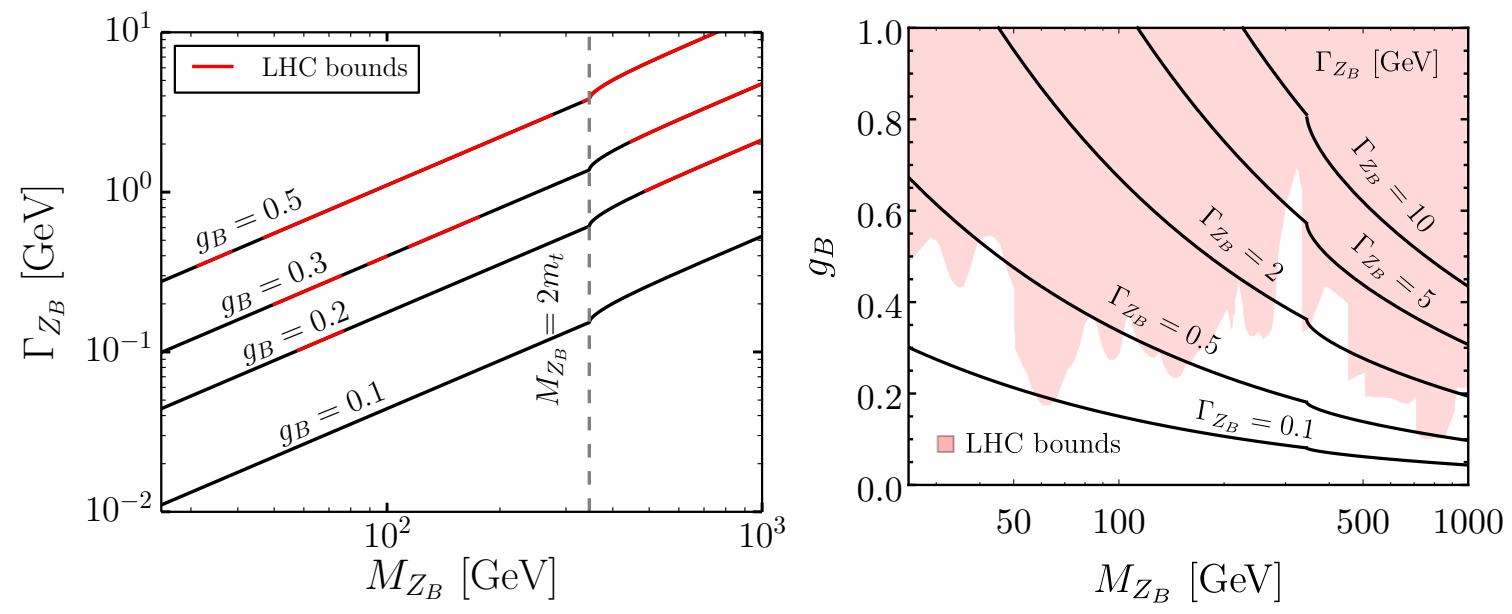

Figure 2. Left panel: decay width of the $Z_{B}$ boson as a function of its mass. The regions highlighted in red are excluded by searches at the LHC. To the right of the dashed vertical line the decay channel $Z_{B} \rightarrow t \bar{t}$ is open. Right panel: contour lines for the decay width of the $Z_{B}$ boson in the $g_{B}$ vs. $M_{Z_{B}}$ plane.

almost any value in the window $25 \mathrm{GeV}<M_{Z_{B}}<1 \mathrm{TeV}$ is allowed. Therefore, there is hope to produce this gauge boson at the LHC with large cross-sections and study its properties.

In the left panel in figure 2 we show the decay width of $Z_{B}$ for different values of the gauge coupling $g_{B}$ as a function of its mass. In red we show the regions that are ruled out by the collider bounds shown in figure 1. From this we can infer which are the allowed values for the decay width of the leptophobic gauge boson. Moreover, with this information of the decay width we can predict the different cross-sections relevant for different collider 


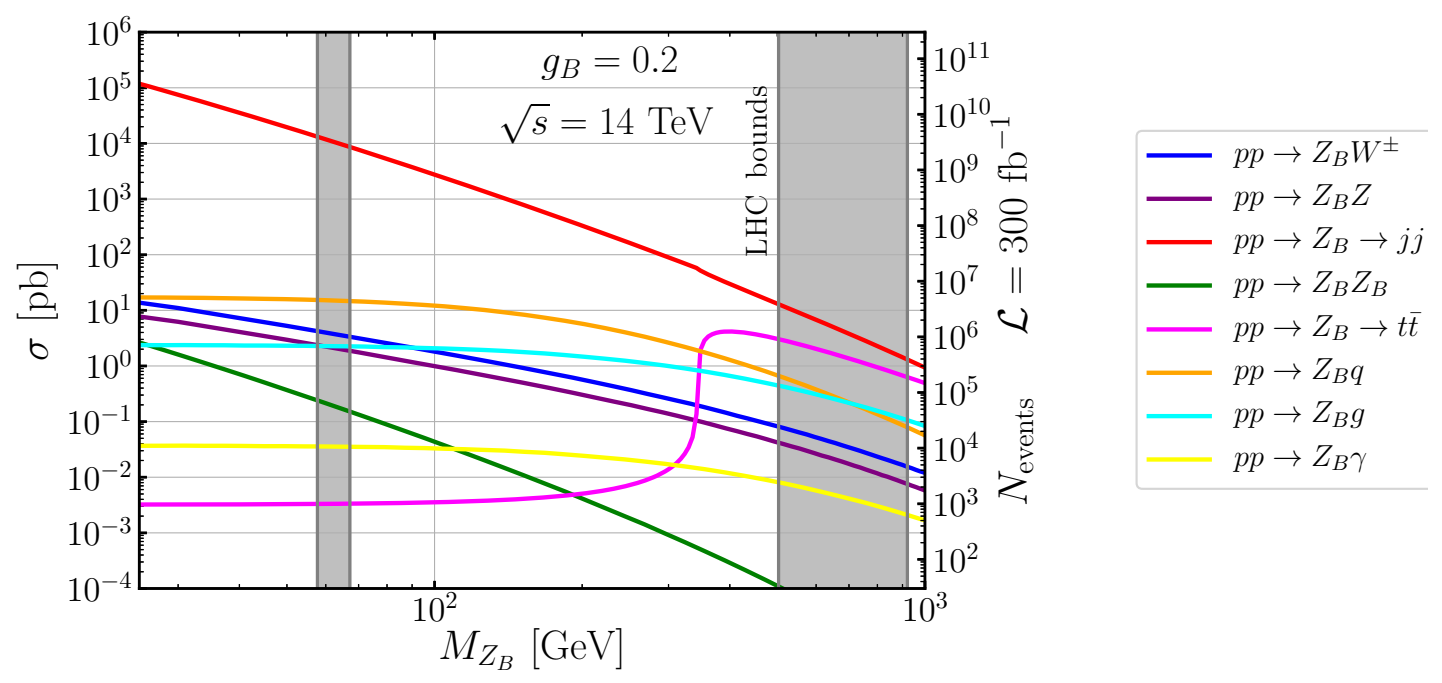

Figure 3. Production cross-sections at the LHC for center-of-mass energy of $14 \mathrm{TeV}$ in units of picobarns, we fixed $g_{B}=0.2$. On the right side of the vertical axis we show the expected number of events assuming $300 \mathrm{fb}^{-1}$ for the integrated luminosity. The regions shaded in gray are excluded by LHC searches for the $Z_{B}$ boson.

searches. In the right panel in figure 2 we present contours of $\Gamma_{Z_{B}}$ in the $g_{B}$ vs. $M_{Z_{B}}$ plane. The region shaded in red is excluded by collider searches of the $Z_{B}$ and we conclude that a $\Gamma_{Z_{B}}$ of order $\mathrm{GeV}$ is already mostly excluded.

In figure 3 we present our results for the production cross-section for different channels that involve at least one $Z_{B}$, fixing the gauge coupling to $g_{B}=0.2$. These results correspond to the LHC with center-of-mass energy of $14 \mathrm{TeV}$ and the number of events shown on the right vertical axis corresponds to an integrated luminosity of $300 \mathrm{fb}^{-1}$. The model has been implemented in FeynRules 2.0 [26] and the cross-sections obtained using MadGraph5aMC@NLO - v2.7.0 [27], we cross-checked our results in a Mathematica notebook and the use of the MSTW2008 [28] set of parton distribution functions. In appendices $\mathrm{A}$ and $\mathrm{B}$ we provide analytic results for all the processes we have considered.

From figure 3 one can see that the dijet cross-section dominates across the plot, and in the region $M_{Z_{B}}>2 M_{t}$ the process $p p \rightarrow Z_{B} \rightarrow t \bar{t}$ can be large as well. The process $p p \rightarrow Z_{B} q$ can be significant, since there is a large contribution from the parton distribution function of the gluon in the initial state. For the $p p \rightarrow Z_{B} \gamma, Z_{B} q$ and $Z_{B} g$ channels we impose the following cuts on the rapidity and the transverse momentum: $|\eta|<2.5$, and $p_{T}>150 \mathrm{GeV}$. These three channels are relevant for searches in the low mass regime.

\section{Exotic decays of the SM-like Higgs}

In extensions of the SM with a leptophobic gauge boson [8-11], its mass generation comes from the vacuum expectation of a new Higgs boson with non-zero baryon number, and hence, the models have two Higgs scalars that can mix with each other. After spontaneous symmetry breaking, the SM-like Higgs will have the following coupling to the leptophobic 

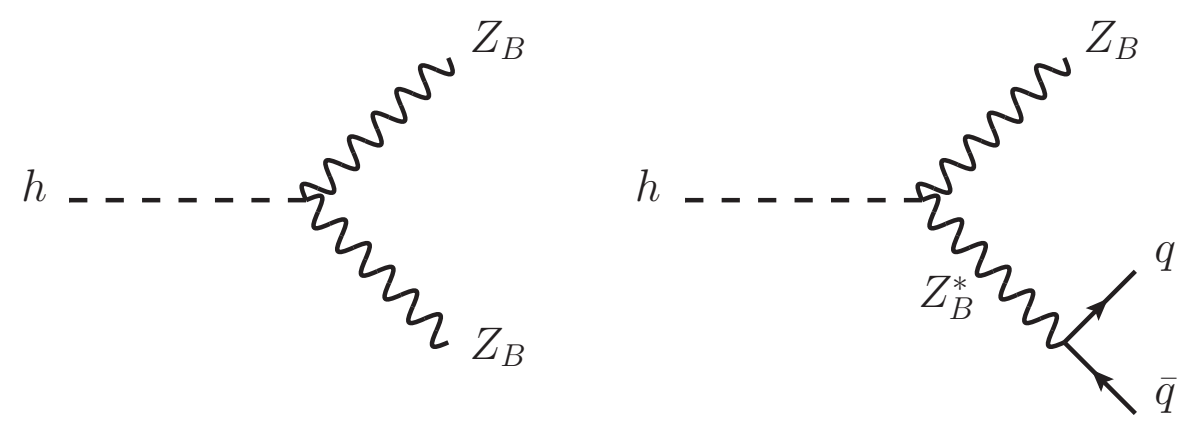

Figure 4. Higgs decays into leptophobic gauge bosons.

$Z_{B}$ gauge boson

$$
h Z_{B}^{\mu} Z_{B}^{\nu}: 2 i \frac{M_{Z_{B}}^{2}}{v_{B}} g^{\mu \nu} \sin \theta_{B}
$$

where $\theta_{B}$ is the mixing angle in the scalar sector, $M_{Z_{B}}=Q_{B} g_{B} v_{B}$ and $Q_{B}$ is the baryon number of the second scalar. Since the leptophobic gauge boson can be light, the SM-like Higgs can have the following decays

$$
h \rightarrow Z_{B} Z_{B}, Z_{B}^{*} Z_{B}
$$

depending on the $Z_{B}$ mass, see figure 4 . In order to calculate these decays one needs to know the coupling between the SM quarks and the $Z_{B}$. Notice that the couplings between the SM-like Higgs and SM particles will scale by a factor $\cos \theta_{B}$. With this information we can calculate the impact of these novel decays of the SM-like Higgs by computing the total Higgs decay width $\Gamma_{h}=\cos ^{2} \theta_{B} \Gamma_{\mathrm{SM}}+\Gamma_{\mathrm{BSM}}$, where in our case $\Gamma_{\mathrm{BSM}}$ corresponds to the decays into two leptophobic gauge bosons.

Collider searches of a new scalar mixing with the SM Higgs combined with measurements of the SM Higgs properties provide constraints on the mixing angle. In our study we take the bound $\sin \theta_{B} \leq 0.3$ [29]. Current LHC measurements of the properties of the SM-like Higgs boson give the following constraint on its branching ratio into BSM particles [30]

$$
\mathrm{BR}(h \rightarrow \mathrm{BSM})<0.34 \quad \text { at } 95 \% \mathrm{CL},
$$

which is obtained assuming the production of the Higgs in the SM. Therefore, we scale the bound by the ratio between the production cross-section for the Higgs in the SM with the one in this model, which is given by $\mathrm{BR}(h \rightarrow \mathrm{BSM})<0.34 \times\left(\sigma_{h}^{\mathrm{SM}} / \sigma_{h}\right)=0.34 / \cos ^{2} \theta_{B}$.

We have computed the two-body and three-body decays and provide analytic expressions in appendix A. In figure 5 we present our results for the branching ratios for the decay channels $h \rightarrow Z_{B} Z_{B}$ and $h \rightarrow Z_{B} q \bar{q}$ of the SM Higgs. The latter includes both, the on-shell and the off-shell contribution from the $Z_{B}$. In the region with $M_{Z_{B}} \leq M_{h} / 2 \approx 62.5 \mathrm{GeV}$ the channel $h \rightarrow Z_{B} Z_{B}$ becomes the dominant decay channel and the Higgs decay width can become of order $\mathrm{GeV}$. In this region the bound on $\mathrm{BR}(h \rightarrow \mathrm{BSM})<0.34$ gives a strong constraint shown by the area shaded in red. The gray band in this figure corresponds to the exclusion bounds from direct searches for the $Z_{B}$ boson at the LHC discussed in section 2 . 

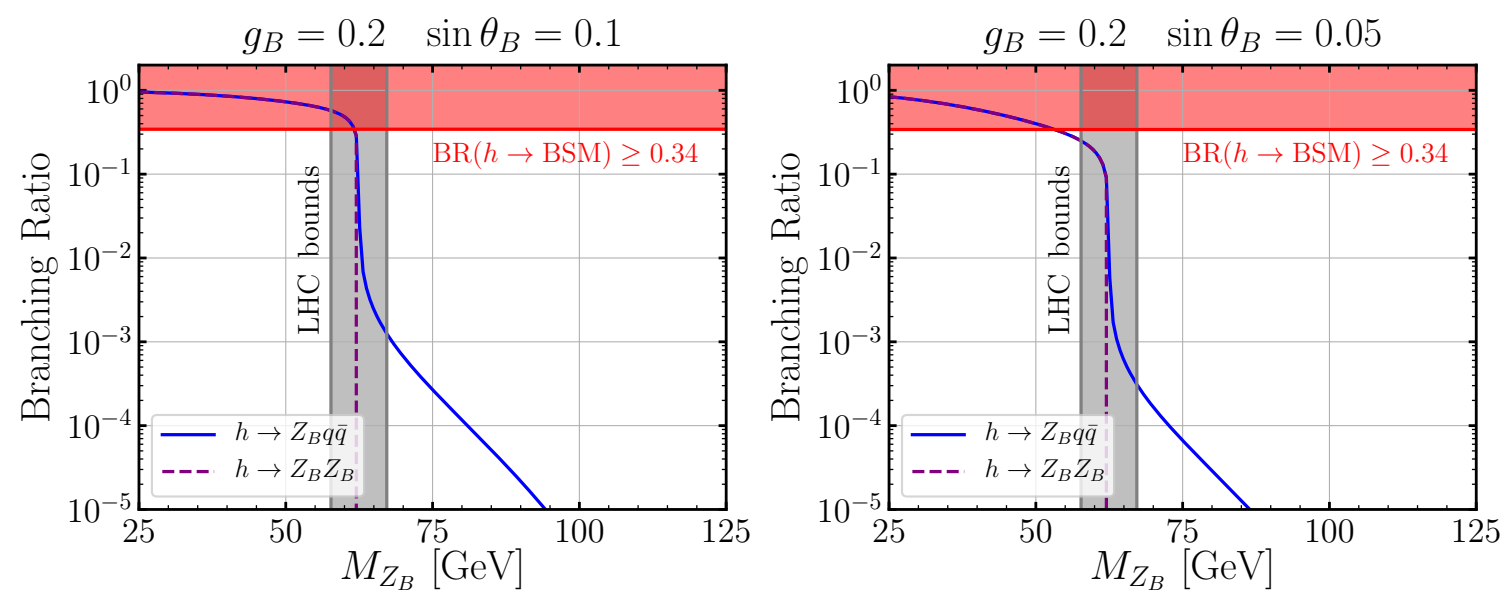

Figure 5. Branching ratios for the channels $h \rightarrow Z_{B} Z_{B}$ and $h \rightarrow Z_{B} Z_{B}^{*} \rightarrow Z_{B} q \bar{q}$. The left (right) panel corresponds to $g_{B}=0.2$ and $\sin \theta_{B}=0.1\left(\sin \theta_{B}=0.05\right)$. The region shaded in red shows the exclusion bounds from the constraint on the SM-like Higgs branching ratio $\mathrm{BR}(h \rightarrow \mathrm{BSM})$ i 0.34. The region shaded in gray corresponds to the exclusion bounds from direct searches for the $Z_{B}$ boson at the LHC.

On the other hand, when $M_{Z_{B}} \geq M_{h} / 2$ the two-body decay is kinematically closed and the three-body decay gives a much smaller contribution to the Higgs width. In this regime, experiments can search for the associated Higgs $Z_{B}$ production to probe the existence of these interactions, as we discuss in the following section.

The experimental bound on the branching ratio of Higgs decays to BSM particles can be translated to the $g_{B}$ vs. $M_{Z_{B}}$ plane. Nevertheless, we note that this bound also depends on the scalar mixing. In figure 6 we present our results for two different mixing angles. For $\sin \theta_{B}=0.1$ this constraint is strong in the region $M_{Z_{B}} \leq M_{h} / 2$ and excludes $g_{B} \gtrsim 0.03$ for $M_{Z_{B}}=25 \mathrm{GeV}$. In order to relax this bound one needs to go to very small mixing angles, $\sin \theta_{B}<0.05$, as shown in the right panel. It is important to emphasize that the SM-like Higgs can have a large branching ratio into two leptophobic gauge bosons in agreement with all current experimental bounds.

\section{Higgs-leptophobic gauge boson associated production}

In the previous section we discussed the possible new Higgs decays due to the existence of a leptophobic gauge boson. In the scenarios where these Higgs decays are not allowed or highly suppressed, one can study the associated production

$$
p p \rightarrow Z_{B}^{*} \rightarrow Z_{B} h,
$$

to test the existence of the new $h-Z_{B}-Z_{B}$ interaction. See figure 8 for the relevant Feynman graph.

The production cross-section for this process is given by eq. (B.4). In figure 7 we show the numerical predictions for the associated production $p p \rightarrow Z_{B}^{*} \rightarrow Z_{B} h$ in the 

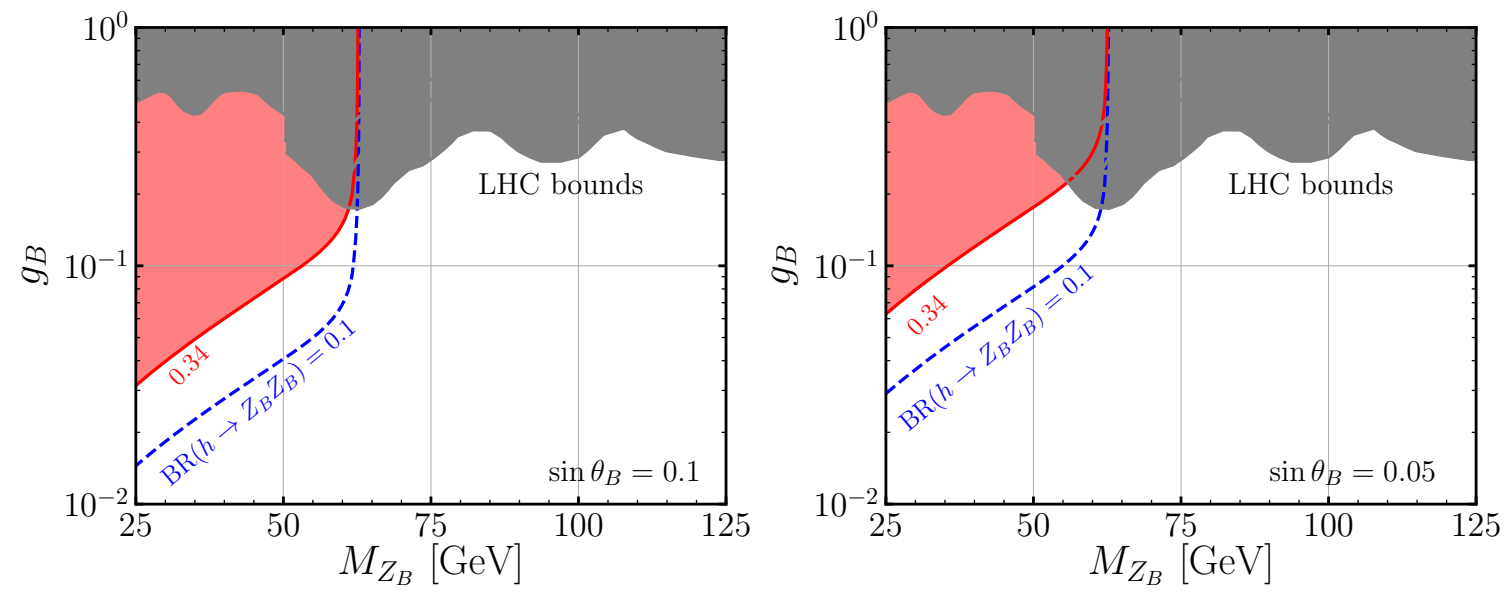

Figure 6. Exclusion bounds in the $g_{B}$ vs. $M_{Z_{B}}$ plane. The region shaded in red shows the exclusion bounds from the constraint on the SM-like Higgs branching ratio $\mathrm{BR}\left(h \rightarrow Z_{B} Z_{B}\right)<0.34$, while the blue dashed line corresponds to $\operatorname{BR}\left(h \rightarrow Z_{B} Z_{B}\right)=0.1$. The region shaded in gray is excluded by searches for the $Z_{B}$ at the LHC. The left (right) panel corresponds to $\sin \theta_{B}=0.1\left(\sin \theta_{B}=0.05\right)$.

$g_{B}-M_{Z_{B}}$ plane, in the maximal mixing scenario where $\sin \theta_{B}=0.3$ and with center-ofmass energy of $\sqrt{s}=14 \mathrm{TeV}$. The region shaded in red is excluded by the experimental bound on the branching ratio of the SM Higgs into BSM particles discussed in the previous section. The different colored dotted regions correspond to the predictions in different ranges: $\sigma<0.1 \mathrm{fb}$ (blue dots), $0.1 \mathrm{fb}<\sigma<1 \mathrm{fb}$ (orange dots), $1 \mathrm{fb}<\sigma<10 \mathrm{fb}$ (yellow dots), $10 \mathrm{fb}<\sigma<100 \mathrm{fb}$ (cyan dots), and $\sigma>100 \mathrm{fb}$ (purple dots). The production crosssection can easily be in the tens of femtobarns which is not too far from the $p p \rightarrow Z h$ crosssection of $990.33 \mathrm{fb}$ in the SM [31]. The region shaded in gray is excluded by the collider bounds discussed in section 2. The associated cross-section is proportional to $\sin ^{2} \theta_{B}$. Therefore, although in the above figure we show only the predictions for $\sin \theta_{B}=0.3$, one can easily find the predictions values for other mixing angles. It is important to mention that the associated production can be significant due to the fact that the gauge coupling can be large and the mass of the leptophobic gauge boson can be below the electroweak scale.

Knowing the possible $h$ and $Z_{B}$ decays we can show the predictions for the number of events at the LHC for the following channels:

$$
\gamma \gamma t \bar{t}, \quad \gamma \gamma b \bar{b}, \quad \gamma \gamma j j, \quad b \bar{b} b \bar{b}, \quad b \bar{b} t \bar{t}, \quad \text { and } \quad b \bar{b} j j .
$$

The number of events for each of these channels is given by

$$
N_{\text {events }}(x \bar{x} y \bar{y})=\mathcal{L} \times \sigma\left(p p \rightarrow Z_{B}^{*} \rightarrow Z_{B} h\right) \times \mathrm{BR}(h \rightarrow x \bar{x}) \times \mathrm{BR}\left(Z_{B} \rightarrow y \bar{y}\right) .
$$

In figure 9 we show the predictions for the expected number of events assuming that the integrated luminosity is $\mathcal{L}=3000 \mathrm{fb}^{-1}$ as planned for the High-Luminosity LHC [32], and using the maximal allowed value for the mixing angle $\sin \theta_{B}=0.3$. The gray regions in figure 9 are excluded by the collider bounds discussed in section 2, while the regions in red are excluded by the experimental bound on the branching ratio of SM Higgs exotic decays. 


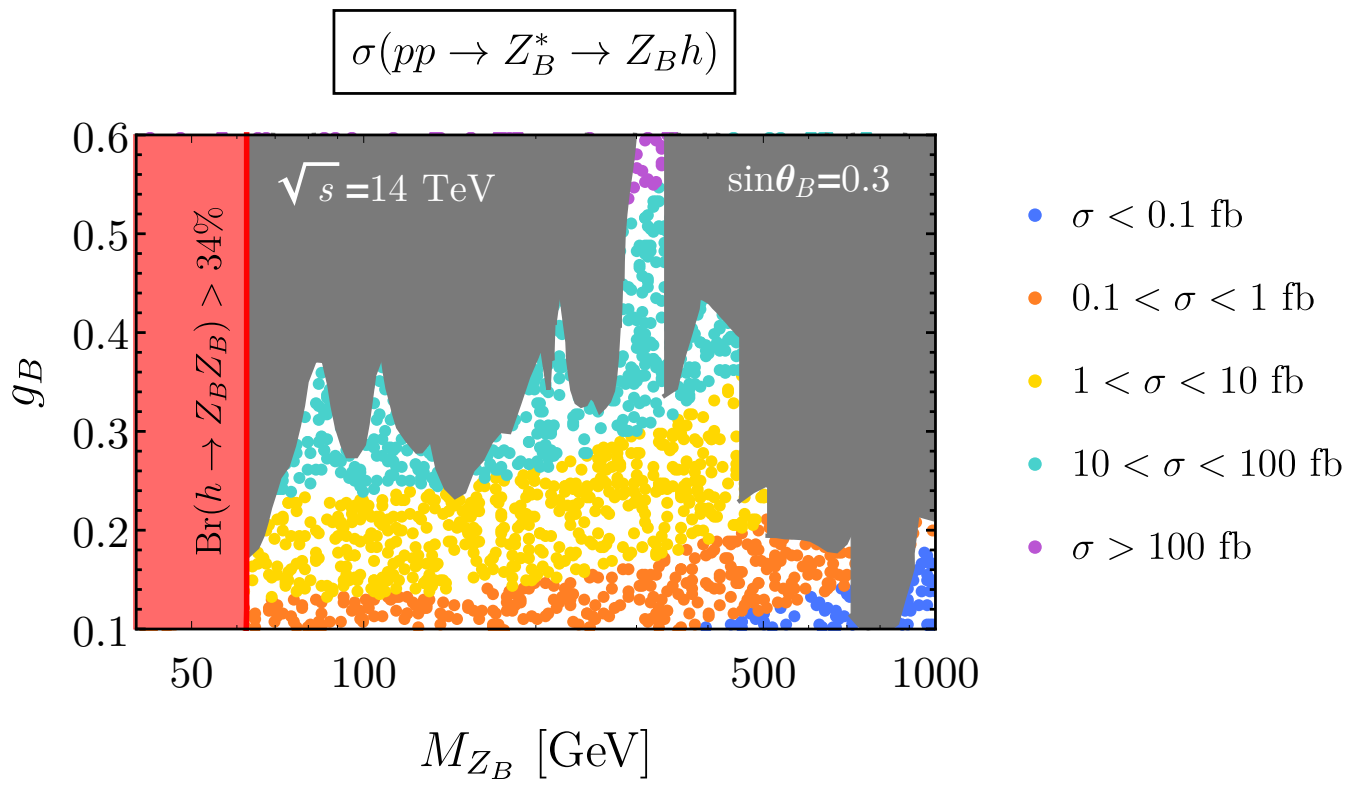

Figure 7. Predictions for the associated production cross-section $p p \rightarrow Z_{B}^{*} \rightarrow Z_{B} h$ at the LHC with center-of-mass energy of $14 \mathrm{TeV}$. The gray region is excluded by the LHC bounds, while the red region is excluded by the bound on the branching ratio of the new Higgs decays. The scalar mixing angle is fixed to $\sin \theta_{B}=0.3$ for this plot.

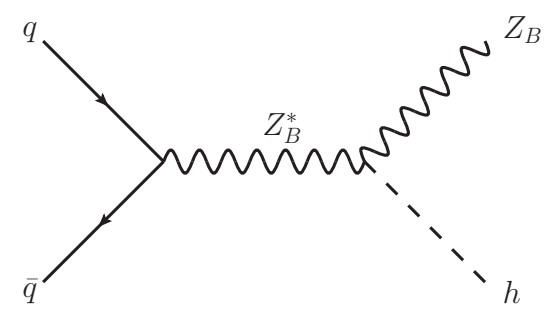

Figure 8. Associated $Z_{B}-h$ production channel.

The $Z h$ associated production has been measured at ATLAS [33] and CMS [34], and a similar technique can be used to make the reconstruction of the processes in figure 9 . However, in our case the $Z_{B}$ decays only to quarks and then the QCD background is more challenging. For example, the largest number of events is for the channel: $p p \rightarrow Z_{B} h \rightarrow$ $j j b \bar{b}$. In this case two $b$ jets should have an invariant mass around the Higgs mass of $125 \mathrm{GeV}$. Furthermore, the large $p_{T}$ of the Higgs or the gauge boson can help discriminate the signal with respect to the background [35]. A dedicated analysis for these signatures is required but it is beyond the scope of this article.

\section{Summary}

The SM Higgs boson can open a doorway to new physics and there is a chance to discover a new sector from the existence of new interactions with the Higgs. In this article, we investigated the possibility that the Higgs can have a new interaction with a leptophobic 

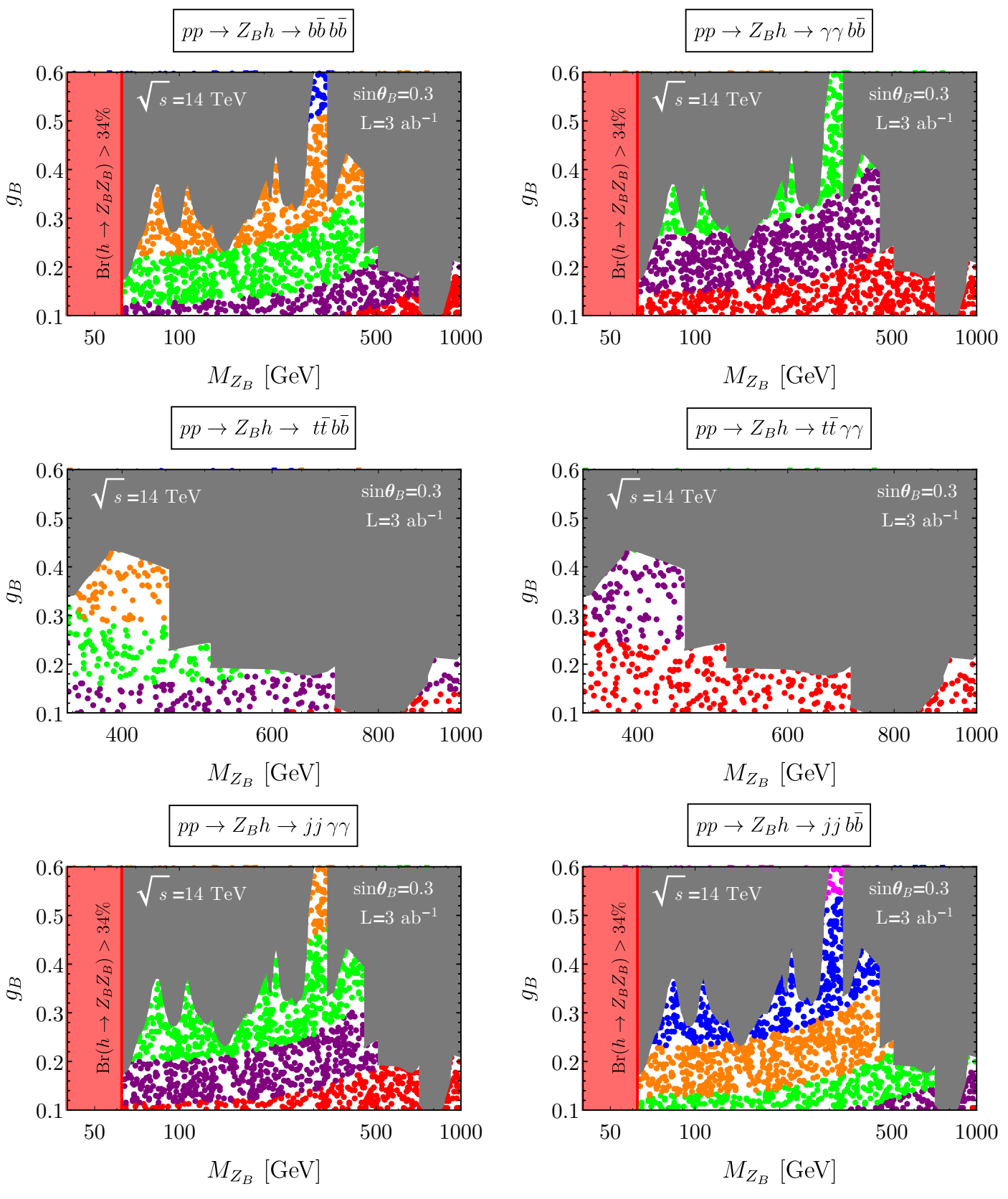

- $N_{\text {events }}>10^{5} \cdot 10^{4}<N_{\text {events }}<10^{5} \cdot 10^{3}<N_{\text {events }}<10^{4}$

- $10^{2}<N_{\text {events }}<10^{3} \cdot 10<N_{\text {events }}<10^{2} \cdot N_{\text {events }}<10$

Figure 9. Predictions for the number of events at the LHC with center-of-mass energy of $14 \mathrm{TeV}$ assuming that the integrated luminosity is $\mathcal{L}=3000 \mathrm{fb}^{-1}$ and using the maximal allowed value for the mixing angle $\sin \theta_{B}=0.3$. We show the number of events for the most relevant channels: $\gamma \gamma t \bar{t}$, $\gamma \gamma b \bar{b}, \gamma \gamma j j, b \bar{b} b \bar{b}, b \bar{b} t \bar{t}$, and $b \bar{b} j j$. The gray region is excluded by the LHC bounds, while the red region is excluded by the bound on the branching ratio of the new Higgs decays. 


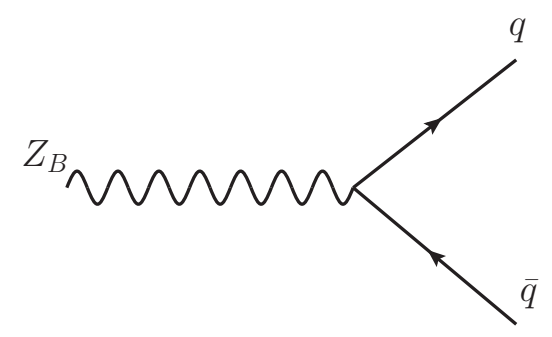

Figure 10. Leptophobic gauge boson decay.

gauge boson. In this scenario, Higgs decays can have a large branching ratio into two leptophobic gauge bosons if they are kinematically allowed. The leptophobic gauge boson can be very light, with mass below the electroweak scale, in agreement with all experimental bounds and without assuming a very small gauge coupling.

In the case where the new Higgs decays are highly suppressed or not allowed, we investigated the associated production of the Higgs and the leptophobic gauge boson at the LHC. We showed that this channel can lead to a large number of events with multiphotons and two quarks, which can be used to probe the existence of the interaction of the Higgs with the new gauge boson. As in the case of the exotic Higgs decays, the production cross-sections can be generically large due to the fact that the leptophobic gauge boson can be light in agreement with all experimental bounds. It is relevant to mention that the possible existence of a leptophobic gauge boson at the low scale tells us that it is possible to have a simple gauge theory where baryon number is a local gauge symmetry [8-11] describing physics below the $\mathrm{TeV}$ scale.

\section{Acknowledgments}

The work of P.F.P. has been supported by the U.S. Department of Energy, Office of Science, Office of High Energy Physics, under Award Number DE-SC0020443. The work of C.M. has been supported in part by Grants No. FPA2014-53631-C2-1-P, No. FPA2017-84445-P, and No. SEV-2014-0398 (AEI/ERDF, EU), and by La Caixa-Severo Ochoa scholarship.

\section{A Decays widths}

- Leptophobic Gauge Boson.

The partial decay width of the leptophobic gauge boson $Z_{B}$ with mass $M_{Z_{B}}$ is given by

$$
\Gamma\left(Z_{B} \rightarrow \bar{q} q\right)=\frac{g_{B}^{2} M_{Z_{B}}}{36 \pi} \sqrt{1-\frac{4 M_{q}^{2}}{M_{Z_{B}}^{2}}}\left(1+\frac{2 M_{q}^{2}}{M_{Z_{B}}^{2}}\right),
$$

where $M_{q}$ is the mass of a given quark. 
- New Higgs Decays.

The width for the new two-body decays, $h \rightarrow Z_{B} Z_{B}$, of the SM Higgs boson is

$$
\Gamma\left(h \rightarrow Z_{B} Z_{B}\right)=\frac{G_{B} M_{h}^{3} \sin ^{2} \theta_{B}}{16 \sqrt{2} \pi} \sqrt{1-4 x}\left(1-4 x+12 x^{2}\right),
$$

with $x=M_{Z_{B}}^{2} / M_{h}^{2}$ and $G_{B}=1 /\left(\sqrt{2} v_{B}^{2}\right)$.

The three-body decay, $h \rightarrow Z_{B}\left(p_{1}\right) q\left(p_{2}\right) \bar{q}\left(p_{3}\right)$, is given by

$$
\Gamma\left(h \rightarrow Z_{B} q \bar{q}\right)=\frac{1}{(2 \pi)^{3}} \frac{1}{32 M_{h}^{3}} \int_{p_{12}^{\min }}^{p_{12}^{\max }} d p_{12} \int_{p_{23}^{\min }}^{p_{23}^{\max }} d p_{23}\left|\bar{A}\left(h \rightarrow \bar{q} q Z_{B}\right)\right|^{2} .
$$

Neglecting the quark masses we have that

$$
\begin{array}{ll}
p_{12}^{\min }=M_{Z_{B}}^{2}, & p_{12}^{\max }=M_{h}^{2}, \\
p_{23}^{\min }=0, & p_{23}^{\max }=\frac{1}{p_{12}}\left(p_{12}-M_{Z_{B}}^{2}\right)\left(M_{h}^{2}-p_{12}\right),
\end{array}
$$

where $p_{i j}=\left(p_{i}+p_{j}\right)^{2}$ and the spin-averaged squared amplitude is given by

$$
\begin{aligned}
\left|\bar{A}\left(h \rightarrow \bar{q} q Z_{B}\right)\right|^{2}= & \frac{8}{3} \frac{g_{B}^{2}}{v_{B}^{2}} \frac{M_{Z_{B}}^{4} \sin ^{2} \theta_{B}}{\left(\left(p_{23}-M_{Z_{B}}^{2}\right)^{2}+M_{Z_{B}}^{2} \Gamma_{Z_{B}}^{2}\right)} \\
& \times\left(p_{23}+\frac{\left(p_{12}-M_{Z_{B}}^{2}\right)\left(M_{h}^{2}-p_{12}-p_{23}\right)}{M_{Z_{B}}^{2}}\right) .
\end{aligned}
$$

\section{B Production cross-sections}

The hadronic production cross-section reads as

$$
\sigma(p p \rightarrow X Y)(s)=\int_{\tau_{0}}^{1} d \tau \frac{d \mathcal{L}_{q \bar{q}}^{p p}}{d \tau} \sigma(q \bar{q} \rightarrow X Y)(\hat{s}),
$$

where $\sigma(q \bar{q} \rightarrow X Y)(\hat{s})$ corresponds to the partonic cross-section and

$$
\frac{d \mathcal{L}_{q \bar{q}}^{p p}}{d \tau}=\int_{\tau}^{1} \frac{d x}{x}\left[f_{q / p}(x, \mu) f_{\bar{q} / p}\left(\frac{\tau}{x}, \mu\right)+f_{q / p}\left(\frac{\tau}{x}, \mu\right) f_{\bar{q} / p}(x, \mu)\right] .
$$

The parameter $\tau=\hat{s} / s$, where $\hat{s}$ is the partonic center-of-mass energy squared, $s$ is the hadronic center-of-mass energy squared, $\tau_{0}=\left(M_{X}+M_{Y}\right)^{2} / s$ is the production threshold, and $\mu$ is the factorization scale. In what follows we give the analytic results for the partonic cross-sections.

- Di-quark production.

The di-quark production cross-section through the leptophobic gauge boson,

$$
\bar{q} q \rightarrow Z_{B}^{*} \rightarrow \bar{q} q
$$




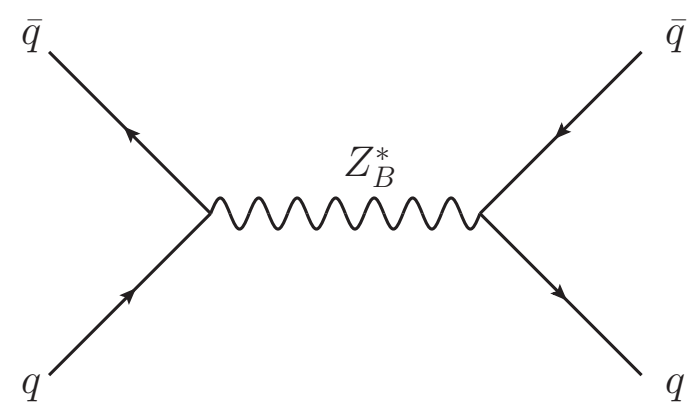

Figure 11. Di-quark production channel.

is given by

$$
\sigma\left(\bar{q} q \rightarrow Z_{B}^{*} \rightarrow \bar{q} q\right)(\hat{s})=\frac{g_{B}^{4} \sqrt{\hat{s}-4 M_{q}^{2}}}{972 \pi \sqrt{\hat{s}}} \frac{\left(2 M_{q}^{2}+\hat{s}\right)}{\left[\left(\hat{s}-M_{Z_{B}}^{2}\right)^{2}+M_{Z_{B}}^{2} \Gamma_{Z_{B}}^{2}\right]},
$$

where we have neglected the quark masses in the initial state.

- Associated production.

The associated $Z_{B}-h$ production,

$$
p p \rightarrow Z_{B}^{*} \rightarrow Z_{B} h,
$$

is relevant to test the existence of the new Higgs interaction with the leptophobic gauge boson.

The cross-section at the partonic level is given by

$$
\begin{aligned}
\sigma\left(\bar{q} q \rightarrow Z_{B}^{*} \rightarrow Z_{B} h\right)(\hat{s})= & \frac{g_{B}^{4} \sin ^{2} \theta_{B}}{144 \pi \hat{s}^{2}} \frac{\left[\hat{s}^{2}-2 \hat{s}\left(M_{Z_{B}}^{2}+M_{h}^{2}\right)+\left(M_{Z_{B}}^{2}-M_{h}^{2}\right)^{2}\right]^{1 / 2}}{\left[\left(\hat{s}-M_{Z_{B}}^{2}\right)^{2}+M_{Z_{B}}^{2} \Gamma_{Z_{B}}^{2}\right]} \\
& \times\left[\hat{s}^{2}+2 \hat{s}\left(5 M_{Z_{B}}^{2}-M_{h}^{2}\right)+\left(M_{Z_{B}}^{2}-M_{h}^{2}\right)^{2}\right],
\end{aligned}
$$

where the $\mathrm{U}(1)_{B}$ charge of the new scalar is taken as $Q_{B}=3$ as in the minimal models [8-11].

- Di-boson production.

Taking the quarks to be massless, the cross-section for the process $q \bar{q} \rightarrow Z_{B} V$ where $V=Z, W^{ \pm}, Z_{B}$ is given by

$$
\begin{aligned}
\sigma\left(q \bar{q} \rightarrow Z_{B} V\right)(s)= & \frac{n g_{B}^{2}\left(C_{V}^{2}+C_{A}^{2}\right)}{108 \pi s^{2}}[-2 \sqrt{f(s)} \\
& \left.+\frac{\left(M_{V}^{2}+M_{Z_{B}}^{2}\right)^{2}+s^{2}}{s-M_{V}^{2}-M_{Z_{B}}^{2}} \log \left(\frac{\sqrt{f(s)}+s-M_{V}^{2}-M_{Z_{B}}^{2}}{\sqrt{f(s)}-s+M_{V}^{2}+M_{Z_{B}}^{2}}\right)\right]
\end{aligned}
$$

where the overall factor $n=1(=1 / 2)$ corresponds to having distinguishable (indistinguishable) particles in the final state,

$$
f(s) \equiv M_{V}^{4}-2 M_{V}^{2}\left(M_{Z_{B}}^{2}+s\right)+\left(M_{Z_{B}}^{2}-s\right)^{2},
$$



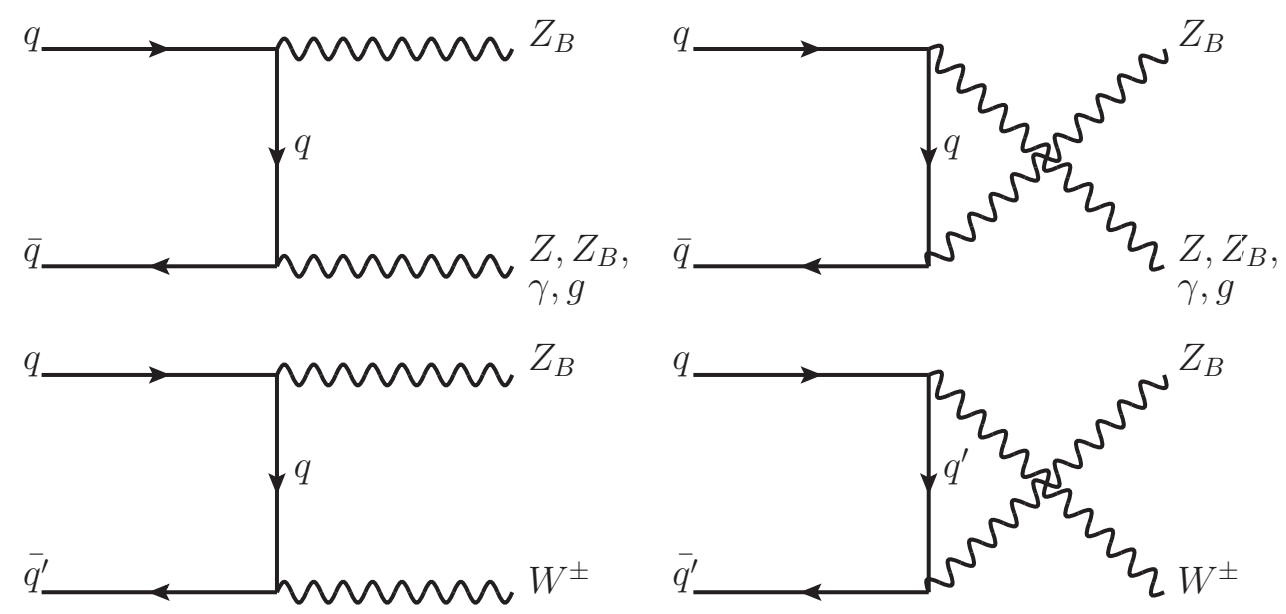

Figure 12. Di-boson production channels.

and the coefficients $C_{V}$ and $C_{A}$ correspond to the vector and axial couplings of the gauge bosons respectively,

$$
\begin{aligned}
Z_{B} Z: C_{V} & =\frac{g_{2}}{\cos \theta_{W}}\left(\frac{1}{2} T_{q}^{3}-Q_{q} \sin ^{2} \theta_{W}\right), C_{A}=-\frac{g_{2}}{2 \cos \theta_{W}} T_{q}^{3} \\
Z_{B} W^{ \pm}: C_{V} & =\frac{g_{2}}{2 \sqrt{2}}, C_{A}=-\frac{g_{2}}{2 \sqrt{2}} \\
Z_{B} Z_{B}: C_{V} & =\frac{g_{B}}{3}, C_{A}=0 .
\end{aligned}
$$

- For the process $q \bar{q} \rightarrow Z_{B} \gamma$ the averaged squared amplitude is given by,

$$
\left|\overline{\mathcal{M}}\left(q \bar{q} \rightarrow Z_{B} \gamma\right)\right|^{2}=\frac{2 e^{2} Q_{q}^{2} g_{B}^{2}\left[M_{Z_{B}}^{4}-2 M_{Z_{B}}^{2} t+s^{2}+2 t(s+t)\right]}{27 t\left(M_{Z_{B}}^{2}-s-t\right)},
$$

where $Q_{q}$ corresponds to the electric charge of the quark. In order to compute the proton-proton cross-section we include the cuts on the transverse momentum and the rapidity of the photon (also gluon and quark) as it is explained in the main text.

- For the process $q \bar{q} \rightarrow Z_{B} g$ we have

$$
\left|\overline{\mathcal{M}}\left(q \bar{q} \rightarrow Z_{B} g\right)\right|^{2}=\frac{8 g_{S}^{2} g_{B}^{2}\left[M_{Z_{B}}^{4}-2 M_{Z_{B}}^{2} t+s^{2}+2 t(s+t)\right]}{81 t\left(M_{Z_{B}}^{2}-s-t\right)},
$$

where $g_{S}$ corresponds to the strong coupling the SM.

- For the process $q g \rightarrow Z_{B} q$, the averaged squared amplitude is given by

$$
\left|\overline{\mathcal{M}}\left(q g \rightarrow Z_{B} q\right)\right|^{2}=\frac{g_{B}^{2} g_{S}^{2}}{27} \frac{M_{Z_{B}}^{4}-2 M_{Z_{B}}^{2} s+2 s^{2}+2 s t+t^{2}}{s\left(s+t-M_{Z_{B}}^{2}\right)},
$$

and we follow the same procedure as above to compute the proton-proton crosssection. 

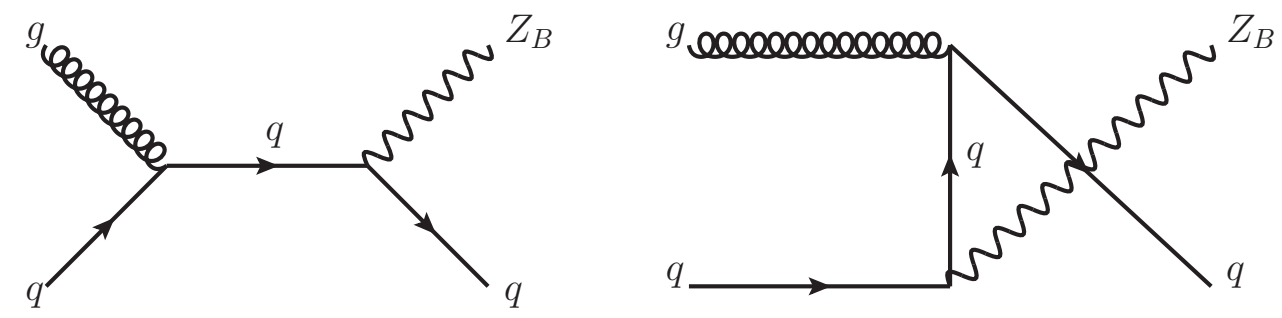

Figure 13. Feyman graphs for $g q \rightarrow Z_{B} q$.

\section{Constraints from kinetic mixing}

In this appendix, we study the kinetic mixing between the $\mathrm{U}(1)_{Y}$ and $\mathrm{U}(1)_{B}$ gauge bosons, see refs. [8-11] for realistic theories where baryon number is a local gauge symmetry. This parameter can be constrained by studying the properties of the $Z$ boson in the SM, see e.g. $[36,37]$. The most general Lagrangian that can be written under the gauge group $\mathrm{SU}(3)_{c} \otimes \mathrm{SU}(2)_{L} \otimes \mathrm{U}(1)_{Y} \otimes \mathrm{U}(1)_{B}$ involving the neutral gauge bosons of the theory is given by

$$
\begin{aligned}
\mathcal{L} \supset & -\frac{1}{4} B_{\mu \nu} B^{\mu \nu}-\frac{1}{2} \operatorname{Tr} W_{\mu \nu} W^{\mu \nu}-\frac{1}{4} B_{\mu \nu}^{\prime} B^{\prime \mu \nu}-\frac{\sin \epsilon}{2} B_{\mu \nu} B^{\prime \mu \nu} \\
& +\frac{1}{8}\left(g_{2} W_{3 \mu}-g_{1} B_{\mu}\right)\left(g_{2} W_{3}^{\mu}-g_{1} B^{\mu}\right) v_{0}^{2}+\frac{1}{2} \mu_{B^{\prime}}^{2} B_{\mu}^{\prime} B^{\prime \mu} \\
& -\sum_{i} \bar{\psi}_{i} \gamma^{\mu}\left[g_{1}\left(Y_{L}^{i} P_{L}+Y_{R}^{i} P_{R}\right) B_{\mu}+g_{2} P_{L} T^{a} W_{a \mu}\right] \psi_{i}+g_{B} \sum_{i} \bar{\psi}_{i} \gamma^{\mu} Q_{B} \psi_{i} B_{\mu}^{\prime},
\end{aligned}
$$

where $Y_{L / R}$ are the hypercharges of the left/right-handed fields interacting with the hypercharge gauge boson $B_{\mu}, Q_{B}=1 / 3$ is the charge of the quarks under the baryon force, $\mu_{B^{\prime}}=3 g_{B} v_{B}$ is the mass term generated after the spontaneously breaking of $\mathrm{U}(1)_{B}$ and $\sin \epsilon$ parametrizes the kinetic mixing between both Abelian gauge bosons $B_{\mu}$ and $B_{\mu}^{\prime}$.

There are different paths to bring the kinetic terms in the first line of eq. (C.1) to an orthonormal form via a non-orthogonal transformation. For convenience, we choose a change of basis that does not modify the well-known relation between the neutral SM gauge bosons, this can be achieved through the following transformation of the $B_{\mu}$ and $B_{\mu}^{\prime}$ fields:

$$
\left(\begin{array}{c}
B_{\mu} \\
B_{\mu}^{\prime}
\end{array}\right) \mapsto\left(\begin{array}{cc}
1 & -\tan \epsilon \\
0 & \sec \epsilon
\end{array}\right)\left(\begin{array}{c}
B_{\mu} \\
B_{\mu}^{\prime}
\end{array}\right)
$$

which renders the kinetic Lagrangian for the gauge bosons orthonormalized and leads to the following mass terms

$$
\begin{aligned}
\mathcal{L} \supset & \frac{1}{8} v_{0}^{2}\left(g_{2} W_{3 \mu}-g_{1}\left(B_{\mu}-\tan \epsilon B_{\mu}^{\prime}\right)\right)\left(g_{2} W_{3 \mu}-g_{1}\left(B^{\mu}-\tan \epsilon B^{\prime \mu}\right)\right) \\
& +\frac{1}{2} \mu_{B^{\prime}}^{2} \sec ^{2} \epsilon B_{\mu}^{\prime} B^{\prime \mu}
\end{aligned}
$$


with the mass matrix in the neutral gauge boson basis $\left(W_{\mu}^{3}, B_{\mu}, B_{\mu}^{\prime}\right)$

$$
M_{0}^{2}=\frac{1}{4}\left(\begin{array}{ccc}
g_{2}^{2} v_{0}^{2} & -g_{1} g_{2} v_{0}^{2} & g_{1} g_{2} \tan \epsilon v_{0}^{2} \\
-g_{1} g_{2} v_{0}^{2} & g_{1}^{2} v_{0}^{2} & -g_{1}^{2} \tan \epsilon v_{0}^{2} \\
g_{1} g_{2} \tan \epsilon v_{0}^{2} & -g_{1}^{2} \tan \epsilon v_{0}^{2} & g_{1}^{2} \tan ^{2} \epsilon v_{0}^{2}+4 \mu_{B^{\prime}}^{2} \sec ^{2} \epsilon
\end{array}\right) .
$$

Now, by rotating the $W_{3}^{\mu}$ and $B^{\mu}$ fields as it is done in the SM,

$$
\left(\begin{array}{c}
B_{\mu} \\
W_{3 \mu}
\end{array}\right)=\left(\begin{array}{cc}
\cos \theta_{W}^{0} & -\sin \theta_{W}^{0} \\
\sin \theta_{W}^{0} & \cos \theta_{W}^{0}
\end{array}\right)\left(\begin{array}{c}
A_{\mu} \\
C_{\mu}
\end{array}\right),
$$

where

$$
\cos \theta_{W}^{0} \equiv \frac{g_{2}}{\sqrt{g_{1}^{2}+g_{2}^{2}}}, \quad \text { and } \quad \sin \theta_{W}^{0} \equiv \frac{g_{1}}{\sqrt{g_{1}^{2}+g_{2}^{2}}},
$$

the photon decouples and we are left with the following mass matrix for the still unphysical neutral gauge bosons $C_{\mu}$ and $B_{\mu}^{\prime}$ :

$$
M_{0}^{2}=\frac{1}{4}\left(\begin{array}{ccc}
0 & 0 & 0 \\
0 & \left(g_{1}^{2}+g_{2}^{2}\right) v_{0}^{2} & \sqrt{g_{1}^{2}+g_{2}^{2}} g_{1} \tan \epsilon v_{0}^{2} \\
0 & \sqrt{g_{1}^{2}+g_{2}^{2}} g_{1} \tan \epsilon v_{0}^{2} & 4 \mu_{B^{\prime}}^{2} \sec ^{2} \epsilon+g_{1}^{2} \tan ^{2} \epsilon v_{0}^{2}
\end{array}\right) .
$$

The above mass matrix defines the angle of the final rotation towards the physical basis,

$$
\begin{aligned}
& C_{\mu}=\cos \xi Z_{\mu}+\sin \xi Z_{B \mu} \\
& B_{\mu}^{\prime}=-\sin \xi Z_{\mu}+\cos \xi Z_{B \mu}
\end{aligned}
$$

given by

$$
\tan 2 \xi=\frac{2 g_{1} \sqrt{g_{1}^{2}+g_{2}^{2}} \tan \epsilon v_{0}^{2}}{4 \mu_{B^{\prime}}^{2} \sec ^{2} \epsilon+g_{1}^{2} \tan ^{2} \epsilon v_{0}^{2}-\left(g_{1}^{2}+g_{2}^{2}\right) v_{0}^{2}},
$$

with the following eigenvalues defining their masses:

$$
\begin{aligned}
M_{A_{\mu}}^{2}= & 0 \\
M_{Z, Z_{B}}^{2}= & \frac{1}{8}\left(g_{1}^{2} \sec ^{2} \epsilon+g_{2}^{2}\right) v_{0}^{2}+\frac{1}{2} \mu_{B^{\prime}}^{2} \sec ^{2} \epsilon \\
& \pm \frac{1}{8} \sqrt{\left(4 \mu_{B^{\prime}}^{2} \sec ^{2} \epsilon+\left(g_{1}^{2} \sec ^{2} \epsilon+g_{2}^{2}\right) v_{0}^{2}\right)^{2}-16\left(g_{1}^{2}+g_{2}^{2}\right) \mu_{B^{\prime}}^{2} v_{0}^{2} \sec ^{2} \epsilon}
\end{aligned}
$$

as expected, in the limit $\epsilon \rightarrow 0$ we recover the original masses in the Lagrangian for $Z$ and $Z_{B}$.

We can now apply the high precision measurement of the $Z$ boson mass to constrain the kinetic mixing parameter, $\sin \epsilon$. The $1 \sigma$ uncertainty in the experimentally measured $Z$ boson mass is [3]

$$
\frac{\Delta M_{Z}}{M_{Z}^{\mathrm{SM}}}=\frac{M_{Z}-M_{Z}^{\mathrm{SM}}}{M_{Z}^{\mathrm{SM}}} \leq \pm 2.3 \times 10^{-5}
$$




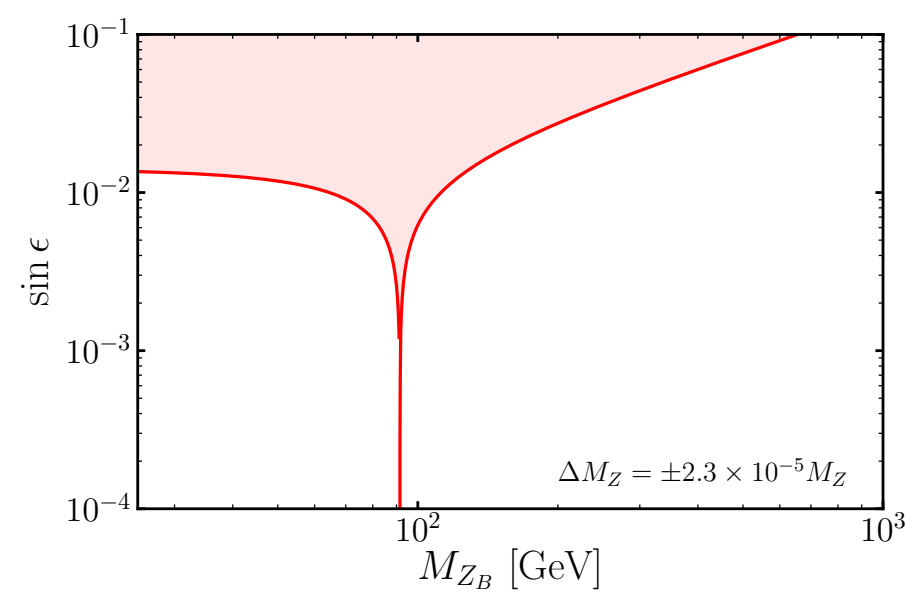

Figure 14. Experimental constraint on the kinetic mixing, $\sin \epsilon$, as a function of the $Z_{B}$ mass. We have used the measurement of the $Z$ boson mass.

and this can be used to constrain the shift induced by the kinetic mixing. In figure 14 we show this constraint in the $M_{Z_{B}}$ vs. $\sin \epsilon$ plane; as can be seen, the kinetic mixing has to be very small and it does not change the main results in our paper. Recently, the CMS [38] and the $\mathrm{LHCb}[39]$ collaborations found stronger constraints for this mixing parameter for $M_{Z_{B}} \leq 200 \mathrm{GeV}$ by searching for the direct production of a new gauge boson.

Open Access. This article is distributed under the terms of the Creative Commons Attribution License (CC-BY 4.0), which permits any use, distribution and reproduction in any medium, provided the original author(s) and source are credited.

\section{References}

[1] ATLAS collaboration, Observation of a new particle in the search for the Standard Model Higgs boson with the ATLAS detector at the LHC, Phys. Lett. B 716 (2012) 1 [arXiv: 1207.7214] [INSPIRE].

[2] CMS collaboration, Observation of a New Boson at a Mass of $125 \mathrm{GeV}$ with the CMS Experiment at the LHC, Phys. Lett. B $\mathbf{7 1 6}$ (2012) 30 [arXiv:1207.7235] [INSPIRE].

[3] Particle Data Group, Review of Particle Physics, Phys. Rev. D 98 (2018) 030001 [INSPIRE].

[4] M. Cepeda et al., Report from Working Group 2: Higgs Physics at the HL-LHC and $H E-L H C$, in Report on the Physics at the HL-LHC, and Perspectives for the HE-LHC, CERN Yellow Reports: Monographs 7, A. Dainese, M. Mangano, A.B. Meyer, A. Nisati, G. Salam and M.A. Vesterinen eds., CERN (2019), pp. 221-584 [arXiv:1902.00134] [INSPIRE].

[5] A. Pais, Remark on baryon conservation, Phys. Rev. D 8 (1973) 1844 [InSPIRE].

[6] R. Foot, G.C. Joshi and H. Lew, Gauged Baryon and Lepton Numbers, Phys. Rev. D 40 (1989) 2487 [INSPIRE].

[7] C.D. Carone and H. Murayama, Realistic models with a light U(1) gauge boson coupled to baryon number, Phys. Rev. D 52 (1995) 484 [hep-ph/9501220] [INSPIRE]. 
[8] P. Fileviez Pérez and M.B. Wise, Baryon and lepton number as local gauge symmetries, Phys. Rev. D 82 (2010) 011901 [Erratum ibid. 82 (2010) 079901] [arXiv: 1002.1754] [INSPIRE].

[9] P. Fileviez Pérez and M.B. Wise, Breaking Local Baryon and Lepton Number at the TeV Scale, JHEP 08 (2011) 068 [arXiv:1106.0343] [INSPIRE].

[10] M. Duerr, P. Fileviez Pérez and M.B. Wise, Gauge Theory for Baryon and Lepton Numbers with Leptoquarks, Phys. Rev. Lett. 110 (2013) 231801 [arXiv:1304.0576] [INSPIRE].

[11] P. Fileviez Pérez, S. Ohmer and H.H. Patel, Minimal Theory for Lepto-Baryons, Phys. Lett. $B 735$ (2014) 283 [arXiv: 1403.8029] [INSPIRE].

[12] P. Fileviez Pérez, New Paradigm for Baryon and Lepton Number Violation, Phys. Rept. 597 (2015) 1 [arXiv: 1501.01886] [INSPIRE].

[13] M. Duerr and P. Fileviez Pérez, Theory for Baryon Number and Dark Matter at the LHC, Phys. Rev. D 91 (2015) 095001 [arXiv:1409.8165] [InSPIRE].

[14] S. Ohmer and H.H. Patel, Leptobaryons as Majorana Dark Matter, Phys. Rev. D 92 (2015) 055020 [arXiv: 1506.00954] [INSPIRE].

[15] M. Duerr, P. Fileviez Pérez and J. Smirnov, Baryonic Higgs at the LHC, JHEP 09 (2017) 093 [arXiv: 1704.03811] [INSPIRE].

[16] P. Fileviez Pérez, E. Golias, R.-H. Li and C. Murgui, Leptophobic Dark Matter and the Baryon Number Violation Scale, Phys. Rev. D 99 (2019) 035009 [arXiv:1810.06646] [INSPIRE].

[17] P. Fileviez Pérez, E. Golias, R.-H. Li, C. Murgui and A.D. Plascencia, Anomaly-free dark matter models, Phys. Rev. D 100 (2019) 015017 [arXiv: 1904.01017] [INSPIRE].

[18] M. Carena, M. Quirós and Y. Zhang, Dark CP-violation and gauged lepton or baryon number for electroweak baryogenesis, Phys. Rev. D 101 (2020) 055014 [arXiv:1908.04818] [INSPIRE].

[19] CMS collaboration, Search for Low-Mass Quark-Antiquark Resonances Produced in Association with a Photon at $\sqrt{s}=13$ TeV, Phys. Rev. Lett. 123 (2019) 231803 [arXiv: 1905.10331] [INSPIRE].

[20] CMS collaboration, Search for narrow resonances in dijet final states at $\sqrt{s}=8 \mathrm{TeV}$ with the novel CMS technique of data scouting, Phys. Rev. Lett. 117 (2016) 031802 [arXiv: 1604.08907] [INSPIRE].

[21] CMS collaboration, Search for narrow resonances in the b-tagged dijet mass spectrum in proton-proton collisions at $\sqrt{s}=8 \mathrm{TeV}$, Phys. Rev. Lett. 120 (2018) 201801 [arXiv: 1802.06149] [INSPIRE].

[22] CMS collaboration, Search for low mass vector resonances decaying into quark-antiquark pairs in proton-proton collisions at $\sqrt{s}=13$ TeV, Phys. Rev. D 100 (2019) 112007 [arXiv: 1909.04114] [INSPIRE].

[23] CMS collaboration, Search for narrow and broad dijet resonances in proton-proton collisions at $\sqrt{s}=13 \mathrm{TeV}$ and constraints on dark matter mediators and other new particles, JHEP 08 (2018) 130 [arXiv: 1806. 00843] [INSPIRE].

[24] CMS collaboration, Search for dijet resonances using events with three jets in proton-proton collisions at $\sqrt{s}=13 \mathrm{TeV}$, Phys. Lett. B 805 (2020) 135448 [arXiv:1911.03761] [INSPIRE]. 
[25] ATLAS collaboration, Search for low-mass dijet resonances using trigger-level jets with the ATLAS detector in pp collisions at $\sqrt{s}=13$ TeV, Phys. Rev. Lett. 121 (2018) 081801 [arXiv: 1804.03496] [INSPIRE].

[26] A. Alloul, N.D. Christensen, C. Degrande, C. Duhr and B. Fuks, FeynRules 2.0 - A complete toolbox for tree-level phenomenology, Comput. Phys. Commun. 185 (2014) 2250 [arXiv:1310.1921] [INSPIRE].

[27] J. Alwall et al., The automated computation of tree-level and next-to-leading order differential cross sections and their matching to parton shower simulations, JHEP 07 (2014) 079 [arXiv: 1405.0301] [INSPIRE].

[28] A.D. Martin, W.J. Stirling, R.S. Thorne and G. Watt, Parton distributions for the LHC, Eur. Phys. J. C 63 (2009) 189 [arXiv:0901.0002] [INSPIRE].

[29] A. Ilnicka, T. Robens and T. Stefaniak, Constraining Extended Scalar Sectors at the LHC and beyond, Mod. Phys. Lett. A 33 (2018) 1830007 [arXiv:1803.03594] [INSPIRE].

[30] ATLAS and CMS collaborations, Measurements of the Higgs boson production and decay rates and constraints on its couplings from a combined ATLAS and CMS analysis of the LHC pp collision data at $\sqrt{s}=7$ and $8 \mathrm{TeV}$, JHEP 08 (2016) 045 [arXiv:1606.02266] [INSPIRE].

[31] LHC Higgs Cross Section Working Group, Handbook of LHC Higgs Cross Sections: 4. Deciphering the Nature of the Higgs Sector, arXiv:1610.07922 [INSPIRE].

[32] G. Apollinari et al., High-Luminosity Large Hadron Collider (HL-LHC), in CERN Yellow Reports: Monographs 4, CERN (2017) [CERN-2017-007-M] [INSPIRE].

[33] ATLAS collaboration, Observation of $H \rightarrow b \bar{b}$ decays and $V H$ production with the ATLAS detector, Phys. Lett. B $\mathbf{7 8 6}$ (2018) 59 [arXiv:1808.08238] [INSPIRE].

[34] CMS collaboration, Evidence for the Higgs boson decay to a bottom quark-antiquark pair, Phys. Lett. B 780 (2018) 501 [arXiv: 1709.07497] [INSPIRE].

[35] J.M. Butterworth, A.R. Davison, M. Rubin and G.P. Salam, Jet substructure as a new Higgs search channel at the LHC, AIP Conf. Proc. 1078 (2009) 189 [arXiv:0809.2530] [InSPIRE].

[36] K.S. Babu, C.F. Kolda and J. March-Russell, Implications of generalized $Z-Z^{\prime}$ mixing, Phys. Rev. D 57 (1998) 6788 [hep-ph/9710441] [InSPIRE].

[37] A. Hook, E. Izaguirre and J.G. Wacker, Model Independent Bounds on Kinetic Mixing, Adv. High Energy Phys. 2011 (2011) 859762 [arXiv:1006.0973] [INSPIRE].

[38] CMS collaboration, Search for a narrow resonance decaying to a pair of muons in proton-proton collisions at $13 \mathrm{TeV}$, CMS-PAS-EXO-19-018 (2019) [INSPIRE].

[39] LHCb collaboration, Search for Dark Photons Produced in 13 TeV pp Collisions, Phys. Rev. Lett. 120 (2018) 061801 [arXiv: 1710.02867] [INSPIRE]. 